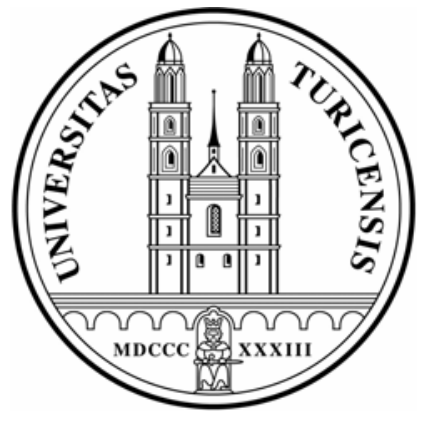

Institute for Empirical Research in Economics

University of Zurich

Working Paper Series

ISSN 1424-0459

Working Paper No. 327

Relative Performance in Bilateral Trade

Christian Ewerhart

June 2007 


\title{
Relative Performance in Bilateral Trade ${ }^{1}$
}

\author{
Christian Ewerhart ${ }^{2}$ \\ University of Zurich
}

First version: April 2007

This version: June 29, 2007

Still preliminary and incomplete!

\begin{abstract}
Concerns for relative performance are integrated into a model of contractual renegotiation in bilateral trade. It is shown that concerns for relative performance do never impede efficient trade. Moreover, conditional on renegotiation to occur, concerns for relative performance tend to mitigate material rent expropriation. However, concerns for relative performance make the occurrence of renegotiation more likely, and may thereby lead to underinvestment even in very optimistic environments. The analysis suggests an explanation for the occurrence of the Druzhba pipeline conflict between Russia and Belarus in January 2007.
\end{abstract}

Keywords: Bilateral trade, incomplete contracting, relative performance, renegotiation, Druzhba pipeline.

JEL classification: D86, L95

\footnotetext{
${ }^{1}$ The idea for this paper has developed following discussions with Ernst Fehr and Oliver Hart. For valuable comments, I am very grateful to Bentley MacLeod and to participants of the 2007 North American Summer Meetings of the Econometric Society. Of course, all remaining errors are my own.

${ }^{2}$ Institute for Empirical Research in Economics, Winterthurerstrasse 30, CH-8006 Zurich, Switzerland.
} 


\section{Introduction}

In long-term bilateral trading relationships, it is not uncommon to observe that original price agreements are renegotiated at a later stage. Such renegotiation should be predicted, for instance, whenever just one party to the contract does not find trade profitable enough at the conditions of the original contract (Hart and Moore, 1988, MacLeod and Malcomson, 1993). A gain in material efficiency may then be possible only if the reluctant party obtains a more attractive contract offer before decisions about trade are made. The consequence of renegotiation to an efficient outcome is that long-term contracts ensure not always the protection of relationship-specific investments. Indeed, renegotiation, unless specifically designed by parties with commitment power (Chung, 1994, Aghion, Dewatripont, and Rey, 1994, Noldeke and Schmidt, 1995), typically leads to an ex-post expropriation of rents, and therefore often to suboptimal levels of relationship-specific investments. ${ }^{3}$

As we will argue in the present paper, this prevalent view on renegotiation in bilateral trade as being triggered solely by material inefficiency may not be general enough to capture all economic applications of general interest. For instance, when prices for oil and gas soared in recent years, the Russian authorities found it increasingly hard to accept that neighboring countries such as Belarus made significant profits from selling inexpensive oil deliveries from Russia at much higher prices to various European countries. This specific conflict culminated in January 2007 in the temporary closure of the important Druzhba pipeline by the Russian government. Our theoretical framework is consistent with the observations made in this case study.

We discuss the trade dispute between Russia and Belarus in more detail in

\footnotetext{
${ }^{3}$ The hold-up inefficiency, i.e., the financial risk associated with relationship-specific investments in uncertain or complex environments, has been described extensively by Williamson $(1975,1985)$. Joskow (1987) offers an insightful discussion of the hold-up problem in the context of the coal industry. The theoretical analysis of the hold-up began with Grout (1984) who studied renegotiation in the context of labor unions. The basic modeling idea has been further developed into various directions, for instance by Tirole (1986) and (in the context of property rights) by Grossman and Hart (1986).
} 
the subsequent section. There, we point out that the renegotiations pushed by Russia since spring 2006 do not correspond to the prevalent understanding in economic theory that renegotiations are aiming at an improvement in material efficiency. Instead we argue that Russia started renegotiations because it was not "content" with a situation in which Belarus made significant profits with subsidized oil imports from Russia. We employ the linear model by Fehr and Schmidt (1999) to study concerns for relative performance in the fundamental incomplete contracting environment that had been proposed by Hart and Moore (1988). For concreteness, we assume that each contractual party compares itself with the respective other party, but in principle, the argument should work analogously for alternative reference points.

We show first that concerns for relative performance alone should not lead to ex-post inefficiency. That is, even though parties care for relative performance and bargaining power is endogenously assigned, the outcome of the renegotiation stage should be be materially efficient. According to this finding, it cannot happen that trading is obstructed just because one party could fear that the other party becomes relatively even wealthier. A second positive result says that the expropriation of material rents is mitigated with concerns for relative performance. This finding should be intuitive. It is more difficult to exploit a party in the weaker bargaining position if that party has concerns for relative performance.

However, all else being equal, renegotiation is more likely to occur with concerns for relative performance. This result is obtained by the combination of two facts. First, there will be scenarios in which the seller, say, finds the price not too low to generate a positive sales margin, but too low in relation to the valuation of the buyer. Indeed, with a utility function that reflects concerns for relative performance, this seller would be credible to unilaterally decide against trade, and so the buyer, interested to get the deal through, would have to make a new contract offer that improves the conditions of the seller sufficiently to make trade attractive even with taking account of the concerns for relative performance. The other fact that is responsible for 
the monotonicity result is that a concern for relative performance can never lead to a situation in which an efficient trading possibility is not exploited by rational agents.

As a consequence of the increased likelihood of renegotiation, incentives for relationship-specific investments may be significantly weakened when parties have concerns for relative performance. We show that even for a very "optimistic" and symmetric environment in which efficiency can be achieved easily between parties with traditional utility specification, the anticipation of renegotiation due to relative performance considerations will make it impossible to implement the first best with a contract that treats the parties in an equitable way.

The rest of the paper is structured as follows. Section 2 gives a brief account of the events related to the Druzhba pipeline closure. In Section 3, we outline the model, and analyze the ex-post stage. Section 4 discusses incentives for investment. Section 5 concludes. The Appendix contains technical proofs.

\section{The Druzhba pipeline dispute}

Connecting the rich oil fields of Western Siberia, the Ural, and the Caspian Sea with refineries located in various continental European countries, the Druzhba pipeline system with a length of about 2,500 miles is usually considered as one of the main energy backbones of Europe. Especially Germany and Poland, but also the Czech Republic, Slovakia, Ukraine, and Hungary rely on the undisturbed working of this connection with its capacity of about 2 million barrels per day. ${ }^{4}$ As can be seen in Figure 1, the pipeline transports crude oil over the territory of Belarus, a former "fraternal socialist ally" at the times of the Soviet Union. Contractual arrangements about prices of oil deliveries date back to times of mutual political support. The name Druzhba means "friendship" in Russian.

\footnotetext{
${ }^{4}$ The average supply during 2006 had been about 1.2 million barrel per day (a barrel corresponds to approximately 159 liters).
} 
Investments into pipelines are clearly of a relationship-specific nature. All parties involved suffer significant losses even from temporary disruptions of supplies. Russia is dependent on the transit through Belarus because of the size of the regular oil deliveries. Redirecting all of the oil normally sent through the transit connection may be difficult if not economically unfeasible. For instance, Ukraine has not a sufficient capacity to replace Belarus as a transit country for oil deliveries. On the other hand, the well-being of the Belarusian economy hinges on the performance of the oil sector. In fact, it has been argued in the media that, to avoid a potential crisis, the country needs to implement economic reforms more urgently before Russian demands can be satisfied.

The Druzhba trade conflict was not an isolated event, but represented an escalation of negotiations about the prices for gas and oil that had been ongoing for some years between the governments of Russia and Belarus. As Gennady Chuffrin, deputy director of the Institute for World Economy and International Relations, put it recently: "Russia doesn't see any returns on its investments in Belarus." ${ }^{5}$ While a deal for gas deliveries and company shares had been struck with much effort in late December of the year 2006, the problem of an appropriate price for oil proved to be more difficult to resolve. The contractual agreement of 1995 was that Russia delivers oil at low prices to Belarus, which in turn should have send 85 percent of its profits back to Russia (which it never did). During spring 2006, the Kremlin tried actively to buy itself into the Belarusian energy sector, targeting in particular the gas monopolist Beltransgaz. With Minsk remaining firm, the dispute between Russia and Belarus assumed a new dimension in late 2006 . After announcements made in December 2006, Russian authorities took two measures in early January of the year 2007 directed at preventing Belarus from re-exporting inexpensive Russian resources to Western Europe at world market prices. First, the state-controlled Gazprom company raised the price

\footnotetext{
${ }^{5}$ More drastic formulations were used in the media. E.g., Global Insight (January 2007) wrote that "the Russian government became so annoyed with being financially milked that it decided to draw the line at all costs."
} 
of its gas to Belarus from USD 46 to USD 100 per 1,000 cubic meter. Second, Russia introduced an export tariff vis-à-vis Belarus of USD 80 per ton of oil. ${ }^{6}$

The government in Minsk sought to impress Russia in return by imposing a transit duty of USD 45 per ton for oil delivered from Russia to Europe. However, Transneft, the Russian oil company operating the Druzhba pipeline, refused to pay this "illegal" fee, arguing that such a fee could not be applied to goods in transit. But then, at least according to Russian sources, Minsk responded by confiscating, from January 6 onwards, oil from the transit pipeline, oil that was destined for European customers. Over the following two days, 79.000 tons out of 91.000 tons had apparently been drawn from the pipeline. On Monday, January 8, Russia, publicly accusing Belarus of stealing oil from the pipeline, halted its oil exports to Central and Western Europe passing through Belarus. In fact, Russian authorities threatened to further disrupt European supplies. These dramatic steps taken by the Russian government brought the conflict to the attention of the general population in Europe (see, e.g., Financial Times, 2007, Business Week Online, 2007).

After three days of uncertainty, in which the behavior of the Russian government was openly condemned by various national authorities and international organizations, the issue was finally settled. Belarusian President Alexander Lukashenko conceded, in a conversation over phone with his Russian counterpart Vladimir Putin, to drop the transit fee on Russian oil. With more than half of the exports from Belarus going to Russia, Putin had a significant bargaining power that might have helped him in the negotiation with Lukashenko. On the evening of Wednesday, January 10, Russia resumed deliveries through the pipeline system. Simultaneously, Belarus began releasing nearly 80,000 tons of oil from the country's depots to its foreign customers,

\footnotetext{
${ }^{6}$ The Russian gas and oil industry is dominated by the two main actors Rosneft and Gasprom. Both companies are majority-owned by the Russian government. Gas and oil are considered by Russia as a strategic industries, i.e., control by foreign companies will not be tolerated, and further control, if not yet established, will be actively sought.
} 
as a compensation of the purportedly tapped oil resources. However, it is generally perceived by insiders that the compromises obtained, both in the case of gas and in the case of oil, will not resolve the trade conflict between Russia and Belarus in the long term.

The Druzhba cutoff was not the first event of this sort. Starting in 2005, the Russian government has persuaded a number of former members of the Soviet Union into accepting higher prices for gas and oil. Georgia, Moldova, and Azerbaijan are among these. A year ago, Russian gas supplies to Europe were interrupted for four days following a price dispute between Russia and Ukraine.

\section{The model}

The case of the transit dispute illustrates the possibility that renegotiation may be the consequence of the unilateral perception by one party to a contract to receive an inappropriate share of the surplus created by the trade. Indeed, with oil prices rising (with some temporary downswings) to higher and higher levels, the Russian government might have observed the comparably sound development of the Belarusian economy in recent years with mixed feelings. This section outlines a model of a trading relationship between two parties that care for relative economic performance.

Our set-up is as follows. Two contractual parties, a seller (S) and a buyer (B), perceive the possibility to enter a potentially profitable trading relationship. The time horizon decomposes into an ex ante and an ex post period, as depicted in Figure 2. Ex ante, parties may agree on some contract for future trade. We will assume a trading technology that allows trading at will (cf. Hart and Moore, 1988), so that a court can only observe whether there has been trade or not. Under this assumption, contracts will be of the form $\left(\widehat{p}_{0}, \widehat{p}_{1}\right)$, where $\widehat{p}_{0}$ is the transfer from the buyer to the seller in case of no trade, and $\widehat{p}_{1}$ is the transfer from the buyer to the seller in the case of trade. This type of contract is consistent with the take-or-pay provisions that have 
been reported for natural gas by Masten and Crocker (1985) and Hubbard and Weiner (1986).

After signing the contract, but still ex ante, seller and buyer each select a respective level of relationship-specific investments $i_{S} \geq 0$ and $i_{B} \geq 0$. Without loss of generality we assume that investments cause no additional costs to either buyer or seller. ${ }^{7}$ Investments are not contractible, but parties can observe mutual investment levels and are consequently symmetrically informed throughout the trading relationship. Throughout the analysis, investments are assumed to be selfish, i.e., the seller's investments do not increase the buyer's valuation of the traded good, nor do the buyer's investments lower the seller's cost of production.

Ex post, the observable, but non-contractible state of the world $\omega \in \Omega$ realizes, where $\Omega$ denotes the set of possible states of the world, and through its realization, a cost function $c\left(i_{S}, \omega\right)$ for the seller, and a value function $v\left(i_{B}, \omega\right)$ for the buyer. The cost is an opportunity cost, and must be paid only if trade occurs. Similarly, the value is understood to be relative to the best alternative, for the buyer, in the ex-post situation. We assume the following simplified renegotiation protocol:

- If both parties prefer trade over no trade at the conditions of the original contract, then there is no renegotiation, and trade occurs.

- If the seller (buyer) prefers trade, and the buyer (seller) prefers no trade at the conditions of the original contract, then the seller (buyer) may send a new signed contract offer $\left(p_{0}, p_{1}\right)$ to the buyer (seller); there is trade when the offer makes the buyer (seller) at least indifferent to the outside option.

- If non of the parties prefers trade over no trade at the conditions of the original contract then parties do not renegotiate, and there is no trade.

\footnotetext{
${ }^{7}$ When actual costs differ from investments, then a straightforward application of the chain rule shows that all results of the paper remain valid in the more general set-up.
} 
As shown by Noldeke und Schmidt (1995), this trading protocol corresponds to a subgame-perfect equilibrium outcome of a fully tractable message game between buyer and seller.

After the completion of the renegotiation stage, the parties decide simultaneously whether to trade or to not trade at the (potentially new) conditions $\left(p_{0}, p_{1}\right)$. The seller's net reward is then given by

$$
x_{S}\left(i_{S}, \omega\right)=p_{q}-q c\left(i_{S}, \omega\right)-i_{S},
$$

where $p_{q}$ denotes the price paid by the buyer to the seller in the ex-post stage. Analogously, the buyer's net reward is given by

$$
x_{B}\left(i_{S}, \omega\right)=q v\left(i_{B}, \omega\right)-p_{q}-i_{B} .
$$

Contractual parties may have concerns for relative performance. For specificity, the utility functions are defined as in Fehr in Schmidt (1999), that is

$$
\begin{aligned}
& u_{S}=x_{S}-\alpha_{S} \max \left\{x_{B}-x_{S}, 0\right\} \\
& u_{B}=x_{B}-\alpha_{B} \max \left\{x_{S}-x_{B}, 0\right\},
\end{aligned}
$$

where we dropped the arguments of the functions $x_{S}$ and $x_{B} \cdot{ }^{8}$ We assume that parameters measuring the extent of disutility from unequal relative performance are given by $\alpha_{S} \in[0 ; 1)$ and $\alpha_{B} \in[0 ; 1)$. Apparently, the limit case $\alpha_{S}=\alpha_{B}=0$ corresponds to the traditional linear specification of the utility function. Note also that, because social preferences are invariant under monotone affine transformations of the utility function, the model would be no more general with a second term for "altruistic" inequity aversion. ${ }^{9}$

\footnotetext{
${ }^{8}$ This assumption is made for convenience only. It will become clear that the results obtained in this paper do not depend on the specific functional form.

${ }^{9}$ For alternative approaches to modeling concerns for relative performance, see Bolton (1991) and Bolton and Ockenfels (2000), for instance.
} 
With traditional quasi-linear utility functions, which are prevalent in main contract-theoretic applications, efficiency is tantamount to maximizing welfare, i.e. to maximizing the sum of individual utility levels. With social utilities, however, utility between individuals is no longer transferable. Only net rewards can be easily transferred via monetary payments between the parties. This does not leave welfare constant because measure of relative performance will react to the shift of wealth from one party to another. To avoid confusion, we will try to take the appropriate amount of care in the definitions of first-best and second-best allocations.

First best. In the first best for parties with concerns for relative performance, gains of trade are shared in an equitable way ex post, and investments ex ante are as if the parties had traditional utility specifications. Formally, denote by $i_{S}^{\mathrm{FB}}$ and $i_{B}^{\mathrm{FB}}$ the investment levels that maximize total surplus in the case of traditional utilities, i.e.,

$$
\left(i_{S}^{\mathrm{FB}}, i_{B}^{\mathrm{FB}}\right)=\arg \max _{\left(i_{S}, i_{B}\right)} E_{\omega}\left[\max \left\{v\left(i_{B}, \omega\right)-c\left(i_{S}, \omega\right) ; 0\right\}-i_{S}-i_{B}\right],
$$

where $E_{\omega}[$.$] denotes the expectated value with respect to the uncertain state$ of the world. The first best between parties with concerns for relative performance now corresponds to the efficient investment ex ante, trade ex-post if and only if $v \geq c$, and a price $p_{q}^{\mathrm{FB}}(\omega)$ that equalizes net rewards, i.e.

$$
p_{q}^{\mathrm{FB}}(\omega)-q c\left(i_{S}, \omega\right)-i_{S}^{\mathrm{FB}}=q v\left(i_{B}, \omega\right)-p_{q}^{\mathrm{FB}}(\omega)-i_{B}^{\mathrm{FB}} .
$$

Rearranging yields

$$
p_{q}^{\mathrm{FB}}(\omega)=q \frac{v\left(i_{B}, \omega\right)+c\left(i_{S}, \omega\right)}{2}+\frac{i_{S}^{\mathrm{FB}}-i_{B}^{\mathrm{FB}}}{2} .
$$

More generally, given investment levels $i_{S}, i_{B}$, and a state $\omega$, we write

$$
p_{q}^{\#}\left(i_{S}, i_{B}, \omega\right)=q \frac{v\left(i_{B}, \omega\right)+c\left(i_{S}, \omega\right)}{2}+\frac{i_{S}-i_{B}}{2}
$$

for the fair ex-post price for trading quantity $q$. The price $p_{q}^{\#}$ is fair in the sense that parties are equally well off in terms of net rewards provided that 
quantity $q$ is traded at price $p_{q}^{\#}$. It can be easily seen that, following a trade of quantity $q$ at price $p_{q}$, the buyer is relatively better off than the seller if and only if $p_{q}<p_{q}^{\#}$ and that the seller is relatively better off than the buyer if and only if $p_{q}<p_{q}^{\#}$.

Ex-post efficiency. Figure 3 shows a renegotiation scenario in which the buyer is relatively better off than the seller in case of no trade at price $\widehat{p}_{0}$, and the seller does not wish to trade at the conditions of the original contract. Material efficiency is achieved in all cases.

Proposition 1. Concerns for relative economic performance do never impede materially efficient trade.

Proof. See the Appendix.

Why can't it happen that concerns for relative performance inhibit ex-post efficient trade? Assume that trade is materially efficient. Then, with "quasilinear" utility, a failure of renegotiation could only happen when the total of the monetary equivalents of the changes in the concerns for relative performance would exceed the gains from trade $v-c$. However, in principle, i.e., ignoring the specific renegotiation protocol for the moment, gains from trade could be shared such that the disutility from inequity would not increase for either buyer or seller. In our linear model, specifically, this could be achieved by sharing the gains from trade equally between buyer and seller. Thus, free (Coasian) bargaining between rational agents should lead to successful renegotiation to the superior utility possibility curve. In contrast to free bargaining, the Hart-Moore-protocol assigns bargaining power as a function of history to either buyer or seller. While this difference affects the distribution of the gains of shares, it should not impair the parties' ability to reach the higher efficiency frontier. ${ }^{10}$

\footnotetext{
${ }^{10}$ This intuition suggests that it should be possible to generalize the argument to more general utility specifications provided that gains in material efficiency shift outwards the inequity-averse utility possibility curve.
} 
The first point to note is that concerns for relative performance make it more difficult for one party to expropriate rents.

Proposition 2. Conditional on renegotiation to occur, concerns for relative economic performance mitigate the expropriation of material rents. That is, given $\left(\widehat{p}_{0}, \widehat{p}_{1}\right), i_{S}, i_{B}$, and $\omega$ such that parties with traditional utility specification renegotiate the initial contract, the renegotiated offer submitted by the buyer (seller) involves a price $p_{1}$ that is weakly higher (weakly lower) for parties with concerns for relative performance than for parties with traditional utility specification.

There are two countervailing effects. On the one hand, the take-it-or-leave-it offer may involve that the party that is supposed to accept the offer will thereby be relatively worse off than the other party. To account for concerns for relative performance, the proposal must in these cases be more generous. The second effect, mitigating the first but never more than compensating it, originates from the fact that higher investments by the party that receives the offer may change that party's perception of what an appropriate price would be.

To see both effects in force, consider a scenario that the buyer makes a new contract proposal to the seller. In this case, the buyer increases the initial price $\widehat{p}_{1}$ to

$$
p_{1}=\widehat{p}_{0}+c+\underbrace{\frac{\alpha_{S}}{1+2 \alpha_{S}}(v-c)}_{\text {envy grant }}-\underbrace{\frac{2 \alpha_{S}}{1+2 \alpha_{S} v} \max \left\{\widehat{p}_{0}-p_{0}^{\#}, 0\right\}}_{\text {idleness tax }},
$$

where $p_{0}^{\#}=\left(i_{S}-i_{B}\right) / 2$, as before. The envy grant reflects the amount by which the buyer has to increase the price to account for the fact that the value of trade happens to be "large" as a consequence of a pure random effect. The contribution effect captures the idea that the differential between the parties' investment levels affects the relative economic position. For instance, if the seller invested less than the buyer, and the ex-ante agreed 
no-trade price would not compensate the buyer in an appropriate way, then rent expropriation will be easier for the buyer.

But under which conditions does renegotiation occur with concerns for relative performance? In the standard analysis, i.e., for $\alpha_{S}=\alpha_{B}=0$, an efficient constellation of cost and value realizations is accompanied with renegotiation is precisely one party prefers trade over no trade at the conditions of the original contract. Thus assume that buyer and seller agreed on a contract $\left(\widehat{p}_{0}, \widehat{p}_{1}\right)$. We shall drop the arguments of cost and value functions in the sequel. Then renegotiation will occur for $v \geq c$ in the standard model if and only if either the buyer prefers trade and the seller prefers no trade at the conditions of the initial contract, i.e., if

$$
v-\widehat{p}_{1}>-\widehat{p}_{0} \text { and } \widehat{p}_{1}-c<\widehat{p}_{0}
$$

or if the buyer prefers no trade and the seller prefers trade at the conditions of the initial contract, i.e., if

$$
v-\widehat{p}_{1}<-\widehat{p}_{0} \text { and } \widehat{p}_{1}-c>\widehat{p}_{0} \text {. }
$$

With concerns for relative performance, conditions (1) and (2) are enriched by envy terms. The buyer wishes to trade at the conditions of the original contract provided that

$$
\begin{gathered}
\left.v-\widehat{p}_{1}-\alpha_{B} \max \left\{\widehat{p}_{1}-c-i_{S}\right)-\left(v-\widehat{p}_{1}-i_{B}\right), 0\right\} \\
\geq-\widehat{p}_{0}-\alpha_{B} \max \left\{\left(\widehat{p}_{0}-i_{S}\right)-\left(-\widehat{p}_{0}-i_{B}\right), 0\right\} .
\end{gathered}
$$

On the other hand, the seller prefers trade provided that

$$
\begin{aligned}
& \widehat{p}_{1}-c-\alpha_{S} \max \left\{\left(v-\widehat{p}_{1}-i_{B}\right)-\left(\widehat{p}_{1}-c-i_{S}\right), 0\right\} \\
& \geq \widehat{p}_{0}-\alpha_{S} \max \left\{\left(-\widehat{p}_{0}-i_{B}\right)-\left(\widehat{p}_{0}-i_{S}\right), 0\right\} .
\end{aligned}
$$

We will show now that the probability of renegotiation is never lower between parties with concerns for relative performance than between parties with traditional utility specification. 
Figure 3 exhibits the indifference curves (between trade and no trade under the conditions of the original contract) for buyer and seller in $(c, v)$-space. Concerns for relative performance are reflected here by kinks of the respective indifference curves at the intersection point with the cross diagonal defined by the equation

$$
\frac{v+c}{2}=\widehat{p}_{1}-p_{0}^{\#}
$$

Indeed, when (3) is satisfied, then

$$
x_{B}-x_{S}=\left(v-\widehat{p}_{1}-i_{B}\right)-\left(\widehat{p}_{1}-c-i_{S}\right)=2\left(\frac{v+c}{2}-\widehat{p}_{1}+p_{0}^{\#}\right)=0 .
$$

so that the net rewards of buyer and seller coincide. When

$$
\frac{v+c}{2}>\widehat{p}_{1}-p_{0}^{\#}
$$

then the buyer is better off, while for the reversed inequality, the seller is better off. The next result assigns a role to inequity aversion as a trigger for re-negotiation.

Proposition 3. Fix $\left(\widehat{p}_{0}, \widehat{p}_{1}\right), i_{S} \geq 0$, and $i_{B} \geq 0$. Then for any state $\omega$ in which renegotiation occurs between parties with traditional utility specification, there is also renegotiation between parties with concerns for relative economic performance.

Thus, economic agents that care about relative performance are more likely to renegotiate any given contract.

The following example scenario captures the logic of the general argument. Assume that the buyer is relatively better off than the seller in the case of no trade at the conditions of the initial contract. Assume also that without envy, the buyer prefers trade, while the seller prefers no trade at the conditions of the initial contract, i.e.,

$$
v-\widehat{p}_{1}>-\widehat{p}_{0} \text { and } \widehat{p}_{1}-c<\widehat{p}_{0} \text {. }
$$


The area in $(c, v)$ space where this occurs is shaded in Figure 4. But in this case, trade can only increase the differential in net rewards because

$$
\left(v-\widehat{p}_{1}-i_{B}\right)-\left(\widehat{p}_{1}-c-i_{S}\right)>\left(-\widehat{p}_{0}-i_{B}\right)-\left(\widehat{p}_{0}-i_{S}\right)>0 .
$$

Thus, in this case trade can only increase envy! This fact has two implications. First, the buyer, who is relatively better off in the case of no trade, remains relatively better off in the case of trade. Thus, the envy terms vanish for the buyer, so the buyer's decision is unaffected by concerns for relative performance in this scenario. For the seller, however, the envy term would increases by trade. Thus, the seller's preference for no trade is even more pronounced in the case that the seller has concerns for relative performance.

In general, the point to note is that the contractual party with traditional utility specification that prefers no trade at the conditions of the original contract can only deteriorate its relative economic performance through trading with a party that prefers trade. Thus, the envy term for the unwilling party can only increase with trade, while the envy term for the willing party can only decrease with trade. Thus, renegotiation is more likely with envy.

When the support of the distribution of cost and value parameters is sufficiently dispersed, then Proposition 3 can be strengthened to a strict comparative statics. Assume for the moment that buyer is relatively better off than the seller in the case of no trade, i.e.,

$$
\widehat{p}_{0} \leq p_{0}^{\#}=\frac{i_{S}-i_{B}}{2}
$$

The seller will prefer trade over no trade at the conditions of the original contract whenever

$$
c \leq \widehat{p}_{1}-\widehat{p}_{0}+2 \alpha_{S}\left(p_{0}^{\#}-\widehat{p}_{0}\right)-2 \alpha_{S}\left\{\frac{v+c}{2}+p_{0}^{\#}-\widehat{p}_{1}\right\}^{+} .
$$

Thus, for

$$
\frac{v+c}{2}<\widehat{p}_{1}-p_{0}^{\#}
$$


the indifference curve of the seller will run parallel to the $v$-axis at value

$$
c=\widehat{p}_{1}-\widehat{p}_{0}+2 \alpha_{S}\left(p_{0}^{\#}-\widehat{p}_{0}\right)
$$

But upwards from its intersection point with the line of equal performance

$$
\frac{v+c}{2}=\widehat{p}_{1}-p_{0}^{\#}
$$

the seller's indifference curve tilts to the left-hand side and crosses the point $\left(\widehat{p}_{1}-\widehat{p}_{0} ; \widehat{p}_{1}-\widehat{p}_{0}\right)$. In contrast, for $\alpha_{S}=0$, the indifference curve would run, over its whole length, parallel to the $v$-axis at value $c=\widehat{p}_{1}-\widehat{p}_{0}$. Thus, there is a wedge-shaped domain where renegotiation occurs between agents that care for relative economic performance, but not between agents with a traditional utility specification. Another area where this appears as well is an upper-left wedge starting at the intersection point of the line of equal performance with the line $v=\widehat{p}_{1}-\widehat{p}_{0}$ (cf. Figure 4$)$.

Proposition 4. Assume that the pair of random variables $(c ; v)$ has support $[\underline{c} ; \bar{c}] \times[\underline{v} ; \bar{v}]$ for constants $0<\underline{c}<\bar{c}$ and $0<\underline{v}<\bar{v}$. Then, for an initial contract $\left(\widehat{p}_{0}, \widehat{p}_{1}\right)$ satisfying

$$
\underline{c}<\widehat{p}_{1}-\widehat{p}_{0}<\bar{v}
$$

and for any pair of relationship-specific investment levels $i_{S} \geq 0$ and $i_{B} \geq 0$, the probability of renegotiation is strictly higher for parties with concerns for relative performance than for parties with traditional utility specification

Propositions 2 and 3 reflect the point that strongly increasing oil prices can induce oil producing countries to start renegotiations with their customers and transit partners, even if the variable costs for production do not change, the efficient quantity is not significantly affected by the price change, and a positive profit margin would result for both parties also in the case of the originally agreed price level. 


\section{Incentives for investment}

It has been shown in the previous section that with concerns for relative performance, rent extraction at the ex-post stage is more difficult. On the other hand, contractual agreements made at the ex ante stage are generally less reliable as a predictor of the trading conditions in the ex post stage. A natural question to ask is therefore whether these effect leads to diminished or to strengthened incentives for relationship-specific investments.

Our final result shows that, from an ex ante perspective, the increased probability of renegotiation may lead to underinvestment even if the economic environment is very "optimistic." For simplicity, we restrict ourselves to a scenario which is "symmetric." Assume that cost and value realizations are independent, and that $c=\underline{c}$ with probability $F\left(i_{S}\right)$, and $c=\bar{c}$ with probability $1-F\left(i_{S}\right)$ for some increasing and differentiable function $F($.$) . Assume$ also that $v=\bar{v}$ with probability $F\left(i_{B}\right)$, and $v=\underline{v}$ with probability $1-F\left(i_{S}\right)$. Assume also that

$$
\underline{c}<\bar{c}<\underline{v}<\bar{v},
$$

with

$$
\bar{v}-\underline{v}=\bar{c}-\underline{c} .
$$

Finally, assume that $\alpha_{S}=\alpha_{B}=\alpha$. There is a unique contract that treats parties symmetrically here, given by

$$
\left(\widehat{p}_{0}, \widehat{p}_{1}\right)=\left(0 ; \frac{\underline{v}+\bar{c}}{2}\right) .
$$

The following example illustrates the inefficiency problem.

Proposition 5. Consider the symmetric scenario outlined above, and assume that

$$
\alpha>\frac{1}{2} \frac{\underline{v}-\bar{c}}{\bar{v}-\underline{v}} .
$$


If then the efficient probabilities $F\left(i_{S}^{\mathrm{FB}}\right)=F\left(i_{B}^{\mathrm{FB}}\right)$ are too low, then the unique contract (7) that treats parties symmetrically leads to inefficient investment levels.

The intuition for this result is the following. Renegotiation is predicted by the model when the realizations of cost and value parameters lead to an inequitable situation. Specifically, when both costs and values are high, then the buyer makes a price concession to the seller, while when both costs and value are low, then the seller makes a a concession to the buyer. This has an impact on the incentives to invest. For the buyer, for instance, the marginal utilitarian return from investment is above the efficient level when costs are low, but the marginal utilitarian return from investment is below the efficient level when costs are high. Thus, when efficiency requires a sufficiently low probability for low costs, then incentives to invest will be too weak for the buyer. Thus, even in this very optimistic scenario, the first-best investment levels are no achievable with a simple contract that treats both parties symmetrically.

\section{Related literature}

There is a significant body of literature that is related to the present paper. This section reviews some closely related contributions.

Klein, Crawford and Alchian (1978) discuss oil pipelines as one of several examples illustrating their conceptual framework. While their discussion focuses on a case with multiple wells and a cluster of refineries, their description of the appropriable parts of the quasi-rents in the pipeline industry should apply without much change to the Druzhba episode. However, their discussion seems to be silent about concerns for relative economic performance between contractual partners that perceive themselves as potential peers on an international political stage.

Masten (1988) considers a rent-seeking model of a long-term trading relationship in which buyer and seller may individually trigger costly re-negotiation 
to a more equitable outcome when unsatisfied with the terms of trade specified in the original contract. Under the assumptions made in the paper, a party to the contract initiates re-negotiation whenever the additional share of the quasi-rents thereby obtainable exceeds the transaction cost of renegotiation for that party. It is shown that the expected sum of transaction costs for buyer and seller is minimized when the ex-ante contract specifies a price that equilibrates the cost-weighted hazards from post-agreement haggling. Section 4 in Masten's paper is particularly interesting because it surveys early evidence on equity provisions in long-term contracts.

In a discussion of Masten's paper, Hellwig (1988) considers several variants of the rent-seeking model, one of which is quite close in spirit to the present analysis. In a nutshell, a buyer and a seller agree to exchange a single unit of an indivisible item at a price $p$. Subsequently, they obtain precise estimates of the buyer's valuation $v>0$ of the item and of the seller's opportunity cost c. The seller may now either deliver the item at the conditions of the original contract or may request re-negotiation. If the seller requests re-negotiation, the buyer's valuation in case of a failure of re-negotiations reduces to $\delta v$, where $0<\delta<1$ is an exogenous parameter. In addition, renegotiation incurs a transaction cost $r^{S} \geq 0$ to the seller. When trade is efficient, then a seller that is willing to trade at the conditions of the original contract would initiate renegotiation for any sufficiently low value of $r^{S}$ and would thereby manage to raise the price by $v(1-\delta) / 2$, where any potential efficiency gain has been split equally between buyer and seller. Thus, as pointed out by Hellwig, the increase in the seller's rent from requesting re-negotiation would be independent of the initial purchase price in this type of model. For our present analysis, we draw the conclusion that hazard equilibriation may not describe relative performance considerations as well as social preferences.

Hart and Moore (2006) study ex-post inefficiencies resulting from behavioral responses to an initial contract. [...to be completed...] 


\section{Conclusion}

In a world with incomplete contracting possibilities, partners to a trading relationship may find themselves in a situation where they would like to rewrite the original contract to reach a mutually beneficial outcome. In this paper, we have argued that the focus on material inefficiency in bilateral trade may not always capture the true motives for ex-post quarreling by a contractual party.

As an example illustrating our claim, we have discussed the recent closure of the Druzhba pipeline that, under normal circumstances, delivers crude oil from Russia to Europe in transit of the territory of Belarus, and secures thereby a significant part of the energy requirements for several developed economies, including Germany and various Central European countries. Based on this example, we have argued that a material disposition may not be the necessary prerequisite for renegotiation to occur, when parties to the trading relationship care also about relative economic performance. Renegotiation may also occur, so we argued, when only one party is unhappy with the idea that the other party ends up with a significant surplus while the own surplus appears much smaller.

It has been shown that, while trade is ex-post materially efficient and conditional on renegotiation, rent expropriation is smoothed, the probability of renegotiation typically increases with concerns for relative performance. In particular, the model captures the effect that one party refuses to trade at the conditions of the original contract, even though it does not posses any bargaining power, and trade would be materially efficient. As a consequence, it may be more difficult to implement efficient incentives to invest between parties with concerns for relative performance. ${ }^{11}$

\footnotetext{
${ }^{11}$ If take-it-or-leave-it offers are not feasible, and trading may occur at several points in time, then the situation changes dramatically. In Ewerhart (2006), we have shown that, for a sufficiently productive technology, concerns for relative performance allow approximating the first best incentives as parties get increasingly patient.
} 


\section{Appendix. Proofs}

Proof of Proposition 1. Consider first the case in which the buyer is relatively better off than the seller in the case of no trade, i.e., $\widehat{p}_{0} \leq p_{0}^{\#}$. We have to show that trade occurs whenever trade is efficient (i.e., $v \geq c$ ). If both buyer and seller prefer trade over no trade at the conditions of the original contract, then the assumed renegotiation protocol implies trade. There are three remaining cases:

Case 1. Only the buyer prefers trade over no trade at the conditions of the original contract. According to our trading protocol, the buyer may then send a new offer to the seller. The only candidate for this offer is the price level $p_{1}$ that makes the seller indifferent between trading at $p_{1}$ and not trading at $\widehat{p}_{0}$. Indeed, any offer lower than $p_{1}$ will be in vain because it is not accepted by the seller. On the other hand, any offer exceeding $p_{1}$ is dominated by a slightly smaller offer. We will check now that it is incentive compatible for the buyer to send the offer $p_{1}$ rather than no offer. To identify $p_{1}$, we show first that $p_{1}$ has the property that the buyer is relatively better off than the seller when trade occurs at price $p_{1}$. To provoke a contradiction, assume that the buyer is not relatively better off than the seller when trade occurs at price $p_{1}$, i.e.,

$$
v-p_{1}-i_{B}<p_{1}-c-i_{S}
$$

Then the price level $p_{1}$ at which the seller is indifferent between no-trade at price $p_{0}$ and under trade at price $p_{1}$ is given implicitly by

$$
p_{1}-c-i_{S}=\widehat{p}_{0}-i_{S}-\alpha_{S}\left(-\widehat{p}_{0}-i_{B}-\left(\widehat{p}_{0}-i_{S}\right)\right) .
$$

Solving for $p_{1}$ yields

$$
p_{1}=\widehat{p}_{0}+c .
$$

Moreover, (9) is tantamount to

$$
\frac{v+c}{2}<p_{1}-p_{0}^{\#}
$$


As $p_{0}^{\#} \geq \widehat{p}_{0}$, this implies

$$
\frac{v+c}{2}<p_{1}-\widehat{p}_{0}
$$

Using (10) yields $v<c$ in contradiction to our efficiency assumption. Thus, we have shown that the buyer is relatively better off than the seller when trade occurs at price $p_{1}$. As a consequence, the price level $p_{1}$ at which the seller is indifferent between no-trade at price $p_{0}$ and under trade at price $p_{1}$ is given implicitly by

$$
\begin{aligned}
& \left.p_{1}-c-i_{S}-\alpha_{S}\left(\left(v-p_{1}-i_{B}\right)-p_{1}-c-i_{S}\right)\right) \\
= & \widehat{p}_{0}-i_{S}-\alpha_{S}\left(-\widehat{p}_{0}-i_{B}-\left(\widehat{p}_{0}-i_{S}\right)\right) .
\end{aligned}
$$

Solving for $p_{1}$ yields

$$
p_{1}=\widehat{p}_{0}+\frac{\left(1+\alpha_{S}\right) c+\alpha_{S} v}{1+2 \alpha_{S}}
$$

We have to check now that it is incentive compatible for the buyer to send this offer $p_{1}$ rather than no offer. The buyer compares her utility from trading at $p_{1}$ with her outside option of not trading and paying $\widehat{p}_{0}$ to the seller. But as

$$
\begin{aligned}
v-p_{1} & =v-\widehat{p}_{0}-\frac{\left(1+\alpha_{S}\right) c+\alpha_{S} v}{1+2 \alpha_{S}} \\
& =\frac{1+\alpha_{S}}{1+2 \alpha_{S}}(v-c)-\widehat{p}_{0} \\
& \geq-\widehat{p}_{0},
\end{aligned}
$$

we have proved that the buyer will make precisely the offer (11). Thus, trades occurs in case 1.

Case 2. Only the seller prefers trade over no trade at the conditions of the original contract. In this case, the seller may send an offer to the buyer. As in case 1 , the only candidate for the offer is the price level $p_{1}$ that makes the buyer indifferent between trade at price $p_{1}$ and no-trade at price $\widehat{p}_{0}$. There 
are two subcases. Assume first that the buyer is relatively better off than the seller when trade occurs at price $p_{1}$, i.e.,

$$
v-p_{1}-i_{B} \geq p_{1}-c-i_{S}
$$

Then $p_{1}$ is implicitly determined by the indifference condition

$$
v-p_{1}-i_{B}=-\widehat{p}_{0}-i_{B}
$$

Thus, in this subcase $p_{1}=\widehat{p}_{0}+v$. Morever, the seller sends this offer if

$$
\begin{aligned}
& p_{1}-c-i_{S}-\alpha_{S}\left(\left(v-p_{1}-i_{B}\right)-\left(p_{1}-c-i_{S}\right)\right) \\
\geq & \widehat{p}_{0}-i_{S}-\alpha_{S}\left(\left(-\widehat{p}_{0}-i_{B}\right)-\left(\widehat{p}_{0}-i_{S}\right)\right),
\end{aligned}
$$

which is equivalent to $v \geq c$. Hence, in the case characterized by (12), there will be trade. Assume now that (12) does not hold, so that the buyer is not relatively better off than the seller when trade occurs at price $p_{1}$. Then $p_{1}$ satisfies the buyer's indifference condition

$$
v-p_{1}-i_{B}-\alpha_{B}\left(\left(p_{1}-c-i_{S}\right)-\left(v-p_{1}-i_{B}\right)\right)=-\widehat{p}_{0}-i_{B} .
$$

Solving for $p_{1}$ yields

$$
p_{1}=\frac{\widehat{p}_{0}+2 \alpha_{B} p_{0}^{\#}}{1+2 \alpha_{B}}+\frac{\left(1+\alpha_{B}\right) v+\alpha_{B} c}{1+2 \alpha_{B}} .
$$

In the considered subcase, it is rational for the seller to send the offer (13) when

$$
p_{1}-c-i_{S} \geq \widehat{p}_{0}-i_{S}-\alpha_{S}\left(\left(-\widehat{p}_{0}-i_{B}\right)-\left(\widehat{p}_{0}-i_{S}\right)\right),
$$

or equivalently, when

$$
p_{1} \geq \widehat{p}_{0}+c-2 \alpha_{S}\left(p_{0}^{\#}-\widehat{p}_{0}\right) .
$$

Using (13), inequality (14) can be seen to be equivalent to

$$
\left(\frac{2 \alpha_{B}}{1+2 \alpha_{B}}+2 \alpha_{S}\right)\left(p_{0}^{\#}-\widehat{p}_{0}\right)+\frac{1+\alpha_{B}}{1+2 \alpha_{B}}(v-c) \geq 0,
$$


which is clearly satisfied under the assumptions made. Thus, again, trade will result.

Case 3. Neither the seller nor the buyer prefers trade over no trade at the conditions of the original contract. This case must be brought to a contradiction. There are again two subcases. We assume first that the buyer is not relatively better off with trade at $\widehat{p}_{1}$ than the seller, i.e.,

$$
v-\widehat{p}_{1}-i_{B}<\widehat{p}_{1}-c-i_{S}
$$

Then, from the seller's preference for no trade at the conditions of the original contract,

$$
\widehat{p}_{1}-c \leq \widehat{p}_{0}-\alpha_{S}\left(\left(-\widehat{p}_{0}-i_{B}\right)-\left(\widehat{p}_{0}-i_{S}\right)\right)
$$

Re-arranging (16) yields

$$
\widehat{p}_{1} \leq \widehat{p}_{0}+c-2 \alpha_{S}\left(p_{0}^{\#}-\widehat{p}_{0}\right)
$$

Similarly, from the buyer's preference for no trade at the conditions of the original contract, expressed by

$$
v-\widehat{p}_{1}-\alpha_{B}\left(\left(\widehat{p}_{1}-c-i_{S}\right)-\left(v-\widehat{p}_{1}-i_{B}\right)\right)<-\widehat{p}_{0},
$$

we obtain

$$
\widehat{p}_{1}>\widehat{p}_{0}+2 \alpha_{B}\left(p_{0}^{\#}-\widehat{p}_{0}\right)+\frac{\alpha_{B} c+\left(1+\alpha_{B}\right) v}{1+2 \alpha_{B}} .
$$

Combining (17) and (18) yields

$$
\frac{1+\alpha_{B}}{1+2 \alpha_{B}}(v-c)+2\left(\alpha_{S}+\alpha_{B}\right)\left(p_{0}^{\#}-\widehat{p}_{0}\right)<0 .
$$

However, comparing (19) with our assumptions $p_{0}^{\#} \geq \widehat{p}_{0}$ and $v \geq c$ yields the desired contradiction. For the other subcase, assume that (15) is not satisfied, i.e., the buyer is relatively better off than the buyer with trade 
at $\widehat{p}_{1}$. Then the seller's strict preference for no trade is expressed by the inequality

$$
\begin{aligned}
& \widehat{p}_{1}-c-\alpha_{S}\left(\left(v-\widehat{p}_{1}-i_{B}\right)-\left(\widehat{p}_{1}-c-i_{S}\right)\right) \\
< & \widehat{p}_{0}-\alpha_{S}\left(\left(-\widehat{p}_{0}-i_{B}\right)-\left(\widehat{p}_{0}-i_{S}\right)\right) .
\end{aligned}
$$

Rewriting yields

$$
\widehat{p}_{1}<\widehat{p}_{0}+\frac{\left(1+\alpha_{S}\right) c+\alpha_{S} v}{1+2 \alpha_{S}}
$$

Combining (20) with the buyer's preference for no trade $\widehat{p}_{1}>\widehat{p}_{0}+v$ yields $v<c$, a contradiction.

This proves the assertion in the case that the buyer is relatively better off with no trade at $\widehat{p}_{0}$. Assume now that the seller is strictly better off than the buyer with trade at $\widehat{p}_{0}$. Consider a mirror ex-post scenario in which the buyer invested $i_{B}^{m}=i_{S}$ and has valuation $v^{m}=\underline{c}+\bar{c}-c$, while the seller invested $i_{S}^{m}=i_{B}$ and has cost $c^{m}=\underline{v}+\bar{v}-v$. Then efficiency in the base scenario, i.e., $v \geq c$, is tantamount to efficiency in the mirror scenario, i.e., $v^{m} \geq c^{m}$. Moreover, for the contract $\left(\widehat{p}_{0}^{m}, \widehat{p}_{1}^{m}\right)=\left(-\widehat{p}_{0},-\widehat{p}_{1}\right)$, the buyer in the mirror scenario is relatively better off with no trade at $\widehat{p}_{0}$. We have already proved that in this case, efficient trade will always take place in the mirror scenario. Thus, efficient trade will also occur in the base scenario. This proves the assertion.

Proof of Proposition 2. Assume that parties have concerns for relative performance. In the case

$$
v \geq c>\widehat{p}_{1}-\widehat{p}_{0}
$$

the buyer makes a proposal to the seller. By Proposition 3, the buyer prefers trade, while the seller prefers no trade. According to the assumption about the renegotiation protocol, the buyer sends a new contract offer $\left(p_{0}, p_{1}\right)$ to the seller. Clearly, the buyer has no advantage of increasing $p_{0}$ above $\widehat{p}_{0}$. 
Similarly, a $p_{0}$ below $\widehat{p}_{0}$ will not find its way to the courts. Thus, without loss of generality, $p_{0}=\widehat{p}_{0}$. Moreover, as the buyer has all the bargaining power in this scenario, the offer will satisfy

$$
\begin{aligned}
& p_{1}-c-\alpha_{S} \max \left\{\left(v-p_{1}-i_{B}\right)-\left(p_{1}-c-i_{S}\right) ; 0\right\} \\
= & \widehat{p}_{0}-\alpha_{S} \max \left\{\left(-\widehat{p}_{0}-i_{B}\right)-\left(\widehat{p}_{0}-i_{S}\right) ; 0\right\} .
\end{aligned}
$$

Rearraging yields

$$
p_{1}-2 \alpha_{S} \max \left\{p_{1}^{\#}-p_{1} ; 0\right\}=\widehat{p}_{0}+c-2 \alpha_{S} \max \left\{p_{0}^{\#}-\widehat{p}_{0} ; 0\right\} .
$$

The left-hand side of (23), considered as a function of $p_{1}$, is strictly increasing, continuous, and unbounded. Hence, there is a unique $p_{1}$ that solves (23). If $p_{1} \geq p_{1}^{\#}$ then

$$
p_{1}=\widehat{p}_{0}+c-2 \alpha_{S} \max \left\{p_{0}^{\#}-\widehat{p}_{0} ; 0\right\} \geq \widehat{p}_{0}+c,
$$

which proves the assertion in this case. Assume now that $p_{1}<p_{1}^{\#}$. Assuming $\widehat{p}_{0} \geq p_{0}^{\#}$ and $p_{1}<p_{1}^{\#}$ yields

$$
p_{1}=\widehat{p}_{0}+c+2 \alpha_{S}\left(p_{1}^{\#}-p_{1}\right)>\widehat{p}_{0}+c .
$$

Finally, if $\widehat{p}_{0}<p_{0}^{\#}$ and $p_{1}<p_{1}^{\#}$, then (23) is tantamount to

$$
p_{1}=\widehat{p}_{0}+\frac{\left(1+\alpha_{S}\right) c+\alpha_{S} v}{1+2 \alpha_{S}}>\widehat{p}_{0}+c .
$$

This proves the assertion for the case $(21)$. The other case in which $\widehat{p}_{1}-\widehat{p}_{0}>$ $v \geq c$ can be dealt with in a completely analogous fashion.

Proof of Proposition 3. Consider a state $\omega$ in which agents with traditional utility specification renegotiate. We will show that also agents with concerns for relative economic performance renegotiate. Assume first that a buyer with traditional utility specification would weakly prefer trade at the conditions of the original contract, while the seller does not wish to trade. 
As trade must be efficient in any state where agents with traditional utility specification enter renegotiation, we have

$$
v \geq c>\widehat{p}_{1}-\widehat{p}_{0}
$$

We will show that the buyer with concerns for relative performance wishes to trade at the conditions of the initial contract, while the seller with concerns for relative performance does not wish to trade. Consider first the buyer's decision. Assume then that the buyer is relatively better off than the seller with no trade at $\widehat{p}_{0}$, i.e., $\widehat{p}_{0} \leq p_{0}^{\#}$. We have to show that

$$
v-\widehat{p}_{1}-\alpha_{B} \max \left\{\left(\widehat{p}_{1}-c-i_{S}\right)-\left(v-\widehat{p}_{1}-i_{B}\right), 0\right\} \geq-\widehat{p}_{0} .
$$

When the maximum term in (25) vanishes, then (25) follows immediately from (24). Otherwise, i.e., when

$$
\left(\widehat{p}_{1}-c-i_{S}\right)-\left(v-\widehat{p}_{1}-i_{B}\right)=2\left(\widehat{p}_{1}-p_{0}^{\#}-\frac{c+v}{2}\right)>0
$$

we have to show that

$$
v-\widehat{p}_{1}-2 \alpha_{B}\left(\widehat{p}_{1}-p_{0}^{\#}-\frac{c+v}{2}\right) \geq-\widehat{p}_{0} .
$$

Re-arranging yields

$$
\frac{\alpha_{B} c+\left(1+\alpha_{B}\right) v}{1+2 \alpha_{B}} \geq \widehat{p}_{1}-\widehat{p}_{0}-2 \alpha_{B}\left(p_{0}^{\#}-\widehat{p}_{0}\right),
$$

which follows from $\widehat{p}_{0} \leq p_{0}^{\#}$ and (24). If the seller is relatively better off with no trade at $\widehat{p}_{0}$, i.e., $\widehat{p}_{0}>p_{0}^{\#}$, then we have to show that

$$
v-\widehat{p}_{1}-2 \alpha_{B} \max \left\{\widehat{p}_{1}-p_{0}^{\#}-\frac{c+v}{2}, 0\right\} \geq-\widehat{p}_{0}-2 \alpha_{S}\left(\widehat{p}_{0}-p_{0}^{\#}\right) .
$$

This inequality is immediate when the maximum term is zero. Under (26), however, (27) is equivalent to

$$
\frac{\alpha_{B} c+\left(1+\alpha_{B}\right) v}{1+2 \alpha_{B}} \geq \widehat{p}_{1}-\widehat{p}_{0}
$$


which follows from (24). Consider now the seller's decision. We have to show that

$$
\widehat{p}_{1}-c-2 \alpha_{S} \max \left\{\frac{c+v}{2}-\widehat{p}_{1}+p_{0}^{\#}, 0\right\}<\widehat{p}_{0}-2 \alpha_{S} \max \left\{p_{0}^{\#}-\widehat{p}_{0}, 0\right\} .
$$

Assume first that

$$
\frac{c+v}{2}-\widehat{p}_{1}+p_{0}^{\#} \leq 0
$$

Rewriting and using (24) yields

$$
p_{0}^{\#}-\widehat{p}_{0} \leq \widehat{p}_{1}-\widehat{p}_{0}-\frac{c+v}{2} \leq 0 .
$$

Thus, if (29) is satisfied, then (28) follows from (24). If, however,

$$
\frac{c+v}{2}-\widehat{p}_{1}+p_{0}^{\#}>0
$$

then (28) reads

$$
\widehat{p}_{1}-\widehat{p}_{0}-\frac{\left(1+\alpha_{S}\right) c+\alpha_{S} v}{\left(1+2 \alpha_{S}\right)}<2 \alpha_{S}\left(p_{0}^{\#}-\widehat{p}_{0}\right)-2 \alpha_{S} \max \left\{p_{0}^{\#}-\widehat{p}_{0}, 0\right\} .
$$

This inequality holds certainly for $p_{0}^{\#} \geq \widehat{p}_{0}$. But also for $p_{0}^{\#}<\widehat{p}_{0},(30)$ implies that

$$
p_{0}^{\#}-\widehat{p}_{0}>\widehat{p}_{1}-\widehat{p}_{0}-\frac{c+v}{2} .
$$

This proves the first part of the Proposition in the case that (24). The other case in which $\widehat{p}_{1}-\widehat{p}_{0}>v \geq c$ is completely analogous and therefore omitted.

Proof of Proposition 4. We will prove the first assertion for the case $\widehat{p}_{0} \leq p_{0}^{\#}$ (the other case $\widehat{p}_{0}>p_{0}^{\#}$ can be dealt with in a completely analogous way). In this case, the buyer is relatively better off than the seller in the no trade scenario. Consider parameters $\varepsilon>\delta>0$, not necessarily small. Define realizations of value and cost parameters

$$
v=\widehat{p}_{1}-\widehat{p}_{0}+\varepsilon \text { and } c=\widehat{p}_{1}-\widehat{p}_{0}-\delta \text {. }
$$


These realizations lie in the support of the ex ante distribution provided that

$$
\varepsilon \leq \bar{v}-\left(\widehat{p}_{1}-\widehat{p}_{0}\right) \text { and } \delta \leq\left(\widehat{p}_{1}-\widehat{p}_{0}\right)-\underline{c} .
$$

With traditional utility specification, both buyer and seller wish to trade at the conditions of the original contract, so there is no renegotiation. With concerns for relative performance, only the buyer wishes to trade when $\delta / \varepsilon$ sufficiently small. To see why, note first that the buyer is relatively better off with trade at $\widehat{p}_{1}$ than the seller. Indeed, a straightforward calculation shows that

$$
\left(v-\widehat{p}_{1}-i_{B}\right)-\left(\widehat{p}_{1}-c-i_{S}\right)=\varepsilon-\delta+2\left(p_{0}^{\#}-\widehat{p}_{0}\right)>0 .
$$

The seller will strictly prefer no trade when

$$
\widehat{p}_{1}-c-\alpha_{S}\left(\varepsilon-\delta+2\left(p_{0}^{\#}-\widehat{p}_{0}\right)\right)<\widehat{p}_{0}-2 \alpha_{S}\left(p_{0}^{\#}-\widehat{p}_{0}\right) .
$$

Rearranging (33) using (31) yields

$$
\delta<\frac{\alpha_{S}}{1+\alpha_{S}} \varepsilon
$$

as a the sufficient condition for the seller's strict preference for no trade at the conditions of the original contract. The buyer, on the other hand, has a strict preference for trade at $\widehat{p}_{1}$ because $v-\widehat{p}_{1} \geq-\widehat{p}_{0}$. Then, renegotiation between agents with concerns for relative performance will take place for realizations satisfying (32) and (34), but not so for agents with a traditional utility specification. By our support assumption (5), this type of situation occurs with positive probability. This proves the first assertion of Proposition 4. To prove the second assertion, assume that $v$ is so large that the seller is unwilling to trade at the conditions of the original contract even for $c=\underline{c}$. From (33), this will be the case provided that

$$
\widehat{p}_{1}-\widehat{p}_{0}-\underline{c}<\alpha_{S}(\varepsilon-\delta) .
$$

Using (34), a sufficient condition for unwillingness to trade on the part of the seller is therefore given by

$$
v \geq \widehat{p}_{1}-\widehat{p}_{0}+\frac{1+\alpha_{S}}{\alpha_{S}}\left(\widehat{p}_{1}-\widehat{p}_{0}-\underline{c}\right) .
$$


This proves the second assertion, and thereby the proposition.

Proof of Proposition 5. To provoke a contradiction, we assume that investment levels resulting from the contract (7) are efficient. We consider the buyer's investment decision $i_{B}$, assuming that the seller chooses $i_{S}^{\mathrm{FB}}$. Denote by $x_{S}(q), x_{B}(q), u_{S}(q)$, and $u_{B}(q)$ the net rewards and utilities of seller and buyer, respectively, when quantity $q$ is traded. If the buyer were to reduce $i_{B}$ marginally below the first-best level $i_{B}^{\mathrm{FB}}$ then

$$
x_{B}(0)=-i_{B}>-i_{B}^{\mathrm{FB}}=-i_{S}^{\mathrm{FB}}=x_{S}(0),
$$

i.e., the buyer would be relatively better off than the seller in the case of no trade at the conditions of the original contract. Hence, with no trade, $u_{B}(0)=-i_{B}$ and

$$
u_{S}(0)=-i_{S}^{\mathrm{FB}}-\alpha_{S}\left(i_{S}^{\mathrm{FB}}-i_{B}\right) .
$$

These are the utility levels down to which, depending on the distribution of the bargaining power, parties will be brought in the renegotiation stage. It is now not difficult to check that the parties will trade at the conditions of the initial contract in states $(\underline{c} ; \bar{v})$ and $(\bar{c} ; \underline{v})$. Indeed, for state $(\bar{c} ; \underline{v})$, by symmetry,

$$
x_{B}(1)=\underline{v}-\widehat{p}_{1}-i_{B}>\underline{v}-\widehat{p}_{1}-i_{B}^{\mathrm{FB}}=\widehat{p}_{1}-\bar{c}-i_{S}^{\mathrm{FB}}=x_{S}(1) .
$$

Thus,

$$
u_{B}(1)=\frac{\underline{v}-\bar{c}}{2}-i_{B}>-i_{B}=u_{B}(0)
$$

and

$$
u_{S}(1)=\frac{\underline{v}-\bar{c}}{2}-i_{B}^{\mathrm{FB}}-\alpha_{S}\left(i_{S}^{\mathrm{FB}}-i_{B}\right)>-i_{B}^{\mathrm{FB}}-\alpha_{S}\left(i_{S}^{\mathrm{FB}}-i_{B}\right)=u_{B}(1),
$$

i.e., both parties would prefer trade over no trade at the conditions of the initial contract. Observing that $(6)$ implies $\widehat{p}_{1}=(\bar{v}+\underline{c}) / 2$, a similar argument can be used to show that trade occurs also in state $(\underline{c} ; \bar{v})$. In state $(\bar{c} ; \bar{v})$,

$$
x_{B}(1)=\widehat{p}_{1}-\bar{c}-i_{B}<\widehat{p}_{1}-\underline{c}-i_{S}^{\mathrm{FB}}=x_{S}(1)
$$


for a sufficiently small deviation $\left(i_{B}^{\mathrm{FB}}-i_{B}\right)$. Hence, in this case,

$$
u_{B}(1)=\frac{\bar{v}-\underline{c}}{2}-i_{B}-\alpha_{B}\left(\left(\frac{\bar{v}-\underline{c}}{2}-i_{S}^{\mathrm{FB}}\right)-\left(\frac{\underline{v}-\underline{c}}{2}-i_{B}\right)\right)>-i_{B}=u_{B}(0)
$$

for a sufficiently small deviation $\left(i_{B}^{\mathrm{FB}}-i_{B}\right)$. Moreover,

$$
u_{S}(1)=\widehat{p}_{1}-\underline{c}-i_{S}^{\mathrm{FB}}>-i_{S}^{\mathrm{FB}}-\alpha_{S}\left(i_{S}^{\mathrm{FB}}-i_{B}\right)=u_{B}(0)
$$

for a sufficiently small deviation $\left(i_{B}^{\mathrm{FB}}-i_{B}\right)$. Thus, the buyer prefers no trade over trade, while the seller prefers trade. A completely analogous analysis of the case $(c ; v)=(\bar{c} ; \underline{v})$ reveals that the in case, the seller prefers no trade, while the buyers prefers trade. Given this expectation about the course of renegotiation, the buyer's expected payoff is given by

$$
\begin{aligned}
E\left[u_{B}\right]= & F\left(i_{B}\right) F\left(i_{S}^{\mathrm{FB}}\right)\left\{\bar{v}-\widehat{p}_{1}-i_{B}\right\} \\
& +F\left(i_{B}\right)\left(1-F\left(i_{S}^{\mathrm{FB}}\right)\right)\left\{\bar{v}-p_{1}-i_{B}\right\} \\
& +\left(1-F\left(i_{B}\right)\right) F\left(i_{S}^{\mathrm{FB}}\right)\left\{-i_{B}\right\} \\
& +\left(1-F\left(i_{B}\right)\right)\left(1-F\left(i_{S}^{\mathrm{FB}}\right)\right)\left\{\underline{v}-\widehat{p}_{1}-i_{B}\right\},
\end{aligned}
$$

where the renegotiated price

$$
p_{1}=\frac{\left(1+\alpha_{S}\right) \bar{c}+\alpha_{S} \bar{v}}{1+2 \alpha_{S}}
$$

in $\operatorname{state}(\bar{c} ; \bar{v})$ follows from the proof of Proposition 2. Collecting terms delivers

$$
\begin{aligned}
E\left[u_{B}\right]= & -i_{B}+F\left(i_{B}\right) F\left(i_{S}^{\mathrm{FB}}\right)\left\{\bar{v}-\widehat{p}_{1}\right\} \\
& +F\left(i_{B}\right)\left(1-F\left(i_{S}^{\mathrm{FB}}\right)\right)\left\{\bar{v}-p_{1}\right\} \\
& +\left(1-F\left(i_{B}\right)\right)\left(1-F\left(i_{S}^{\mathrm{FB}}\right)\right)\left\{\underline{v}-\widehat{p}_{1}\right\} .
\end{aligned}
$$

The necessary first-order condition for the buyer using (7) implies that

$$
f\left(i_{B}\right)\left(F\left(i_{S}^{\mathrm{FB}}\right)\left\{\bar{v}-\widehat{p}_{1}\right\}+\left(1-F\left(i_{S}^{\mathrm{FB}}\right)\right)\left\{\bar{v}-\underline{v}+\left(p_{1}-\widehat{p}_{1}\right)\right\} \geq 1,\right.
$$

where $f($.$) denotes the derivative of F($.$) . Thus incentives are efficient only$ if

$$
\left(F\left(i_{S}^{\mathrm{FB}}\right)\left\{\bar{v}-\widehat{p}_{1}\right\}+\left(1-F\left(i_{S}^{\mathrm{FB}}\right)\right)\left\{\bar{v}-\underline{v}+\left(p_{1}-\widehat{p}_{1}\right)\right\} \geq \bar{v}-\underline{v} .\right.
$$


Re-arranging yields

$$
F\left(i_{S}^{\mathrm{FB}}\right) \geq \frac{p_{1}-\widehat{p}_{1}}{\left(\underline{v}-\widehat{p}_{1}\right)+\left(p_{1}-\widehat{p}_{1}\right)}
$$

or equivalently, if

$$
F\left(i_{S}^{\mathrm{FB}}\right) \geq 1-\frac{1+2 \alpha}{2 \alpha} \frac{\underline{v}-\bar{c}}{\bar{v}-\underline{v}} .
$$

The right-hand side of inequality (35) is positive because of assumption (8). Thus, if the efficient probability of the good state is too small, then concerns for relative performance imply that efficient incentive levels cannot constitute an equilibrium. 


\section{References}

Aghion, P., Dewatripont, M., and P. Rey, 1994, Renegotiation Design with Unverifiable Information, Econometrica 62, 257-82.

Bolton, G., 1991, A Comparative Model of Bargaining: Theory and Evidence, American Economic Review 81, 1096-136.

Bolton, G., and A. Ockenfels, 2000, ERC: A Theory of Equity, Reciprocity, and Competition, American Economic Review 90, 166-193.

Business Week Online, 2007, Russia's Latest Energy Scuffle, by Bush, Jason, January 10.

Chung, T. Y., 1991, Incomplete Contracts, Specific Investments, and Risk Sharing, Review of Economic Studies 58, 1031-42.

Ewerhart, C., 2006, The Effect of Sunk Costs on the Outcome of AlternatingOffers Bargaining between Inequity-Averse Agents, Schmalenbach Business Review 58, special issue, April, 184-203.

Fehr, E., and K. Schmidt, 1999, A Theory of Fairness, Competition and Cooperation, Quarterly Journal of Economics 114, 817-68.

Financial Times, 2007, Russia Shuts Oil Pipeline to Europe, January 9.

Grossman, S., and O. Hart, 1986, The Costs and Benefits of Ownership, A Theory of Lateral and Vertical Integration, Journal of Political Economy 94, 691-719.

Grout, P. A., 1984, Investment and Wages in the Absence of Binding Contracts: A Nash Bargaining Approach, Econometrica 52, 449-60.

Hellwig, M., 1988, Equity, Opportunism, and the Design of Contractual Relations, Comment, Journal of Institutional and Theoretical Economics 144, 200-207. 
Joskow, P., 1987, Contract Duration and Relationship-Specific Investments: Empirical Evidence from Coal Markets, American Economic Review 77, March, 168-85.

Hart, O., and J. Moore, 1988, Incomplete Contracts and Renegotiation, Econometrica 56, 755-85.

Hart, O., and J. Moore, 2006, Contracts as Reference Points, mimeo, Harvard Law School.

Hubbard, R. G., and Weiner, R. J., 1986, Regulation and Long-term Contracting in US Natural Gas Markets, Journal of Industrial Economics 35(1), 71-79.

Klein, B., Crawford, R., and A. Alchian, 1978, Vertical Integration, Appropriable Rents, and the Competitive Contracting Process, Journal of Law and Economics 21, 297-326.

MacLeod, W. B., and J. M. Malcomson, 1993, Investment, Holdup, and the Form of Market Contracts, American Economic Review 83, 811-37.

Masten, S. E., and Crocker, K. J., 1985, Efficient Adaptation in Long-Term Contracts: Take-or-Pay Provisions for Natural Gas, American Economic Review 75, December, 1083-93.

Masten, S. E., 1985, Equity, Opportunism, and the Design of Contractual Relations, Journal of Institutional and Theoretical Economics 144, 180-195.

Noldeke, G., and Schmidt, K. M., 1995, Option Contracts and Renegotiation: A Solution to the Hold-up Problem, Rand Journal of Economics 26(2), Summer, 163-79.

Tirole, J., 1986, Procurement and Renegotiation, Journal of Political Economy 94, 235-59. 
Williamson, O., 1975, Markets and Hierarchies: Analysis and Antitrust Implications, Free Press, New York.

Williamson, O., 1985, The Economic Institutions of Capitalism, Free Press, New York. 

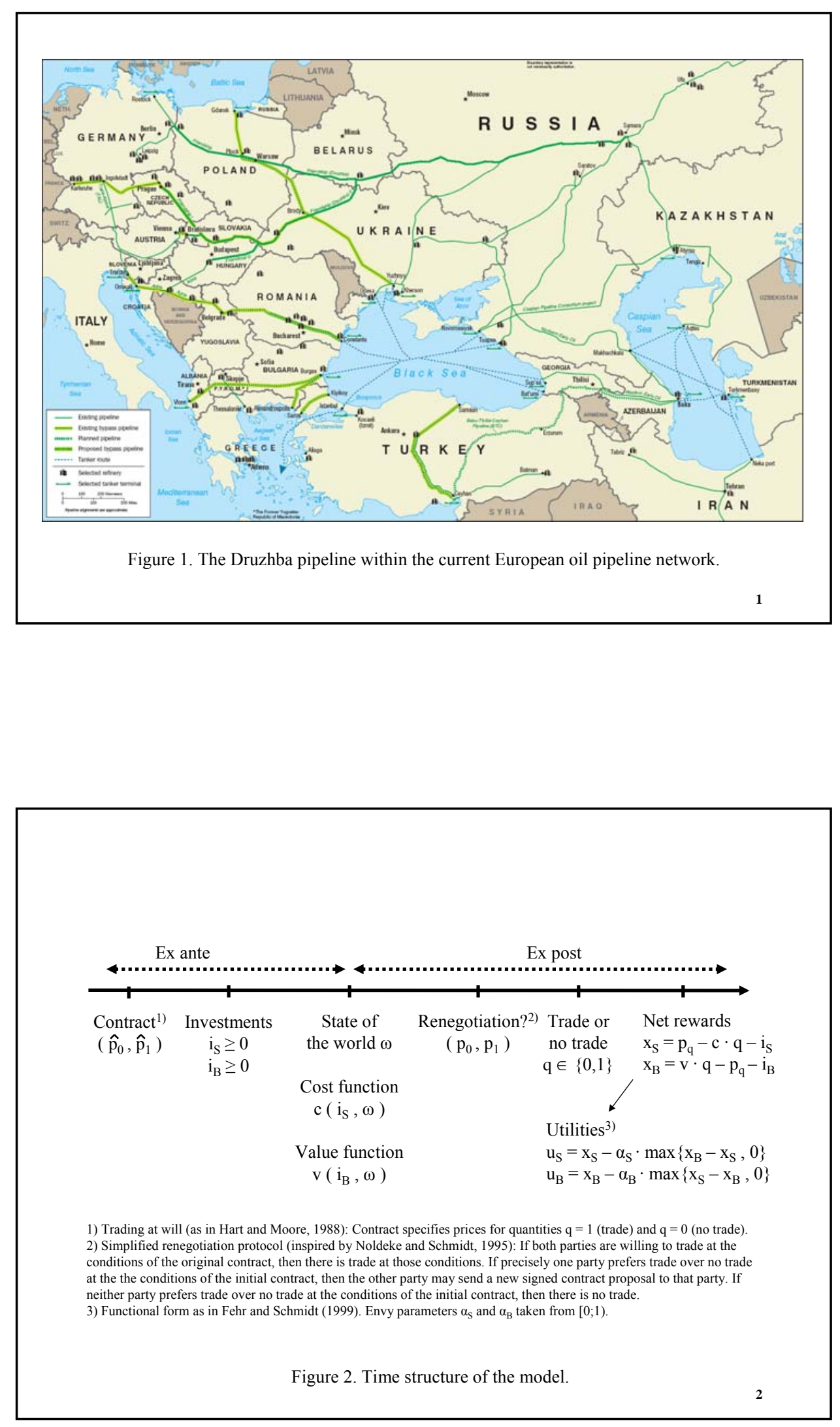

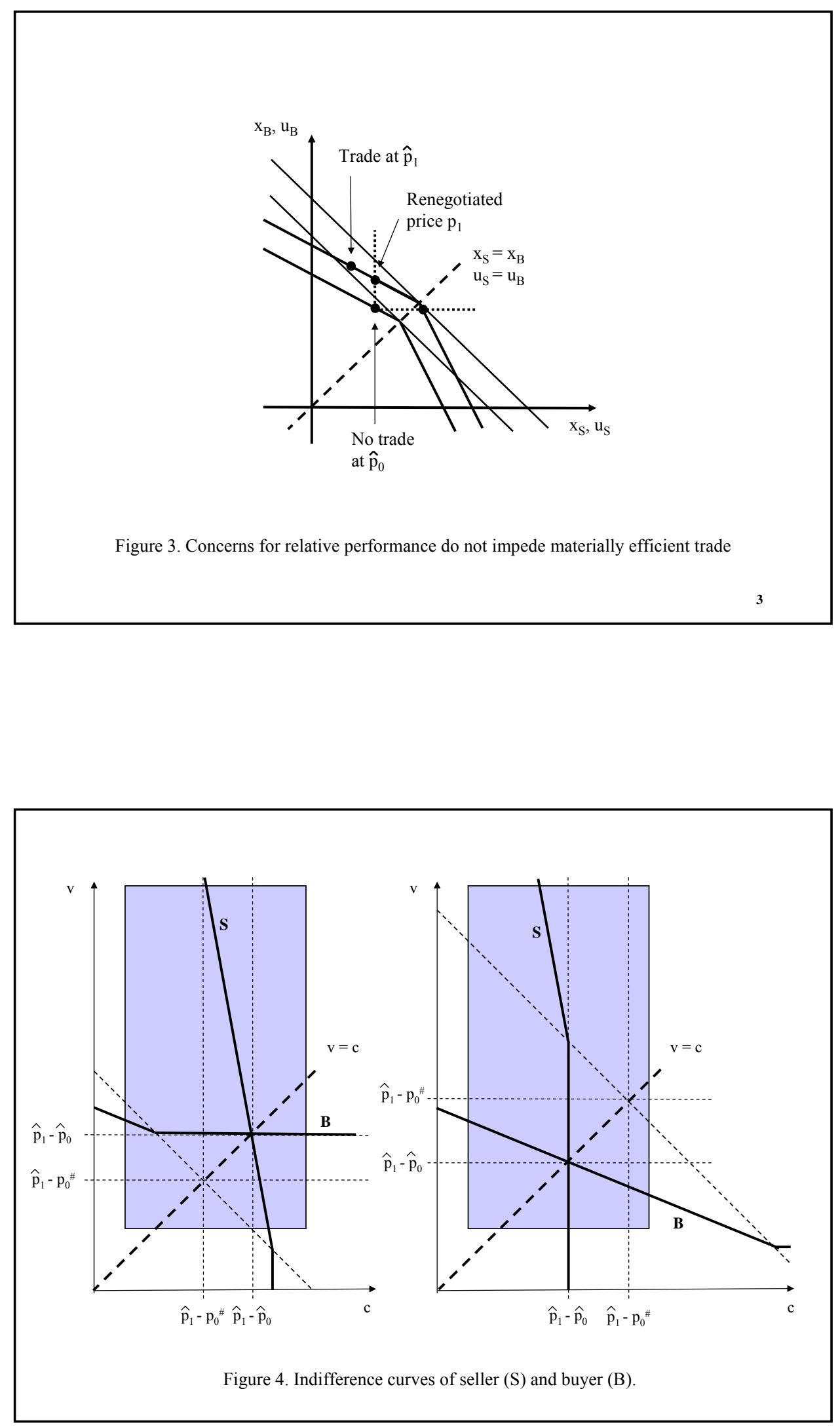


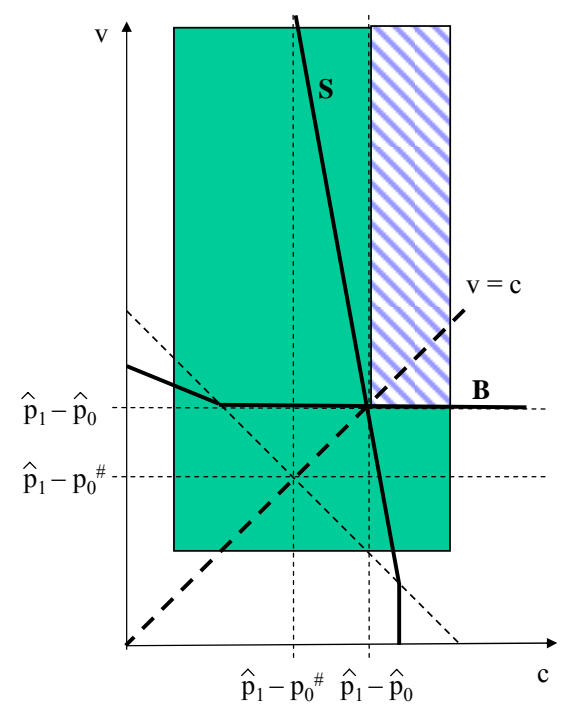

Figure 5. Concerns for relative performance make renegotiation more likely

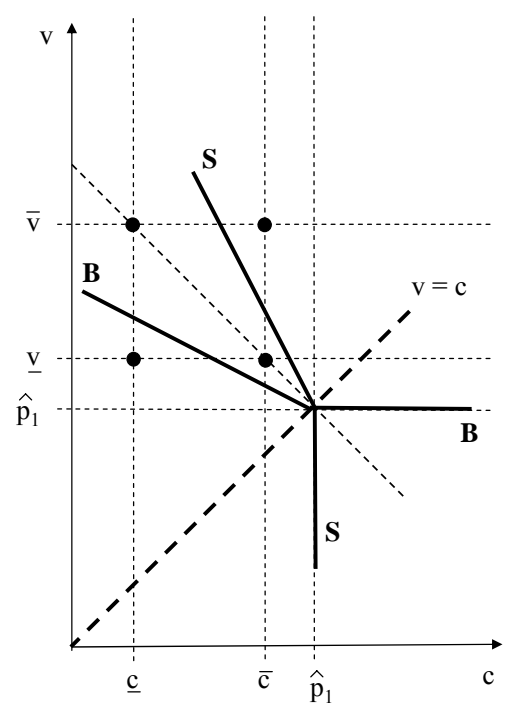

Figure 6. Concerns for relative performance may therefore cause underinvestment even in a very optimistic scenario 